\title{
Quantitative Analysis of Floristic Composition, Biological Spectrum and Leaf Spectrum of a Sacred Grove in Jhargram District, West Bengal, India
}

\author{
Uday Kumar Sen*, Ram Kumar Bhakat \\ Laboratory of Ecology and Taxonomy, Department of Botany and Forestry, Vidyasagar University \\ Vidyasagar University Rd, Rangamati, Midnapore, West Bengal 721102, India \\ *Email: uudaysen@gmail.com
}

\begin{abstract}
Sacred Groves are tracts of virgin forests, left untouched and protected by local people, because of culture and religious beliefs. These tracts are remnants of the once-dominant flora, reservoirs of the rich biodiversity, and the last refuge for preserving the rich indigenous culture and traditions. For these reasons, the biological and leaf spectra, as well as the conservation status of the current sacred grove vegetation, Maa Mongalmoyee Than (MMT) in Jhargram district of West Bengal, India, have been studied. Data were collected during different seasons. The floristic list is taxonomically arranged based on clade, order, and family. In addition, photographs of some common, locally uncommon, endemic and valuable plant species within the sacred grove were taken. The herbarium sheets were then described by matching properly annotated materials available at the Herbarium Section of Vidyasagar University as well as the Botanical Survey of India. The results of floristic studies showed 217 MMT's angiosperm species, belonging to 196 genera, distributed under 59 families of 27 orders. Furthermore, Poales (13.82\%) and Fabaceae (12.44\%) are the dominant order and family, respectively, in terms of species population. Meanwhile, the biological spectrum showed the grove enjoys a "thero-chamaephyte" phytoclimate form, as well as a comparatively undisturbed status, being a sacred grove. Also, the preservation of germplasm within the grove is based on traditional belief in the social system.
\end{abstract}

Keywords: APG IV; biodiversity conservation; biological spectrum; leaf spectra; sacred grove

Article History: Received 12 September 2020; Received in revised form 30 October 2020; Accepted 23 November 2020; Available online 30 December 2020

How to Cite This Article: Sen UK, Bhakat RK. 2020. Quantitative analysis of floristic composition, biological spectrum and leaf spectrum of a Sacred Grove in Jhargram District, West Bengal, India. Biogenesis: Jurnal Ilmiah Biologi. vol 8(2): 157-171. doi: https://doi.org/10.24252/bio.v8i2.16407.

\section{INTRODUCTION}

According to Khan et al. (2008), a classic example of culturally responsive communitybased natural small-scale resource management, are sacred groves. These are small patches of forests devoted to deities and ancestral spirits, with socio-cultural, spiritual and political significance for the indigenous communities caring for them. All around the world, sacred groves have also provided a rich bio-diverse ecosystem, preserved culture, created awareness for bio-diversity conservation, and fostered mutual respect between people and nature within these communities, over the centuries (Verschuuren et al., 2012). Meanwhile, international organizations, including the UNESCO, the IUCN and the WWF are attracting increasing interest in the role of these natural sacred sites. The IUCN treats sacred groves as natural religious places, in addition to areas recognized as sacred by indigenous and traditional communities, or as places of worship and remembrance by religions or faiths. Natural sacred sites are highly diverse in biology and culture, and are the oldest protected areas in the world.

A study by Hughes \& Chandran (1998) stated immemorial sacred groves have been preserved and revered by numerous people, in the name of indigenous God or Goddess, within Africa, Asia, Autro-Pacific region, North and South America, as well as Europe, throughout history, while Kent (2013) reported the existence of forest areas where supernatural entities were claimed to live and immoral practices, including tree felling, wood poaching, plant or leaf collection, hunting, domestic animal grazing, were forbidden.

India has the highest number of sacred groves in the entire world, estimated at over 100000 (Khan et al., 2008), as a result of high ethno-cultural diversity. These groves occur in many regions, with a variety of cultural practices (Rath \& Ormsby, 2020). Indigenous communities across India have protected forest patches as indigenous sacred groves, for generations (Bhagwat \& Rutte, 2006). 
However, these forests, along with the sacred groves are currently disappearing due to cultural change, industrialization, agricultural land encroachment, and exotic weed invasions.

Berkes (2017) reported sacred forests as islands of biodiversity protecting a large number of plant and animal species, including some rare, endangered and endemic taxa, although called by different names in different states of India, and controlled by local people for various reasons. Generally, sacred groves are repositories and nurseries of many local tribal and other folk medicines, and these are the original sources gradually entering modern medicine, after careful screening (Sen, 2018). This article therefore, provides an insight on how human beliefs, norms, social practices, and ethics, help conserve plants in a tribal area of Jhargram district in West Bengal, through sacred groves.
The sacred grove studied (latitude $22^{\circ} 35^{\prime} 51.39^{\prime \prime} \mathrm{N}-22^{\circ} 35^{\prime} 50.27^{\prime \prime} \mathrm{N}$ and longitude $86^{\circ} 51^{\prime} 57.82^{\prime \prime} \mathrm{E}-86^{\circ} \quad 51^{\prime} 57.39^{\prime \prime} \mathrm{E}$; average altitude 103 masl) consists of mixed deciduous, semi-deciduous and evergreen flora species, the present sacred grove, is popularly known as MMT (named the adjacent village as well as the presiding deity, Mongalmoyee). According to Fig. 1, the grove neighbours Malabati village, under Binpur II block, in Jhargram district, along the Jharkhand state's border, and is bordered on the north by Purulia and Bankura districts, on the east by the district of West Midnapore. In addition, the grove has widespread borders with the state of Orissa on the south, and with Jharkhand state, on the south. The region is also extremely humid and tropical. Temperatures are as high as $46^{\circ} \mathrm{C}$, during the hot, dry days of May and June, and as low as $8^{\circ} \mathrm{C}$ in the chilly nights of December

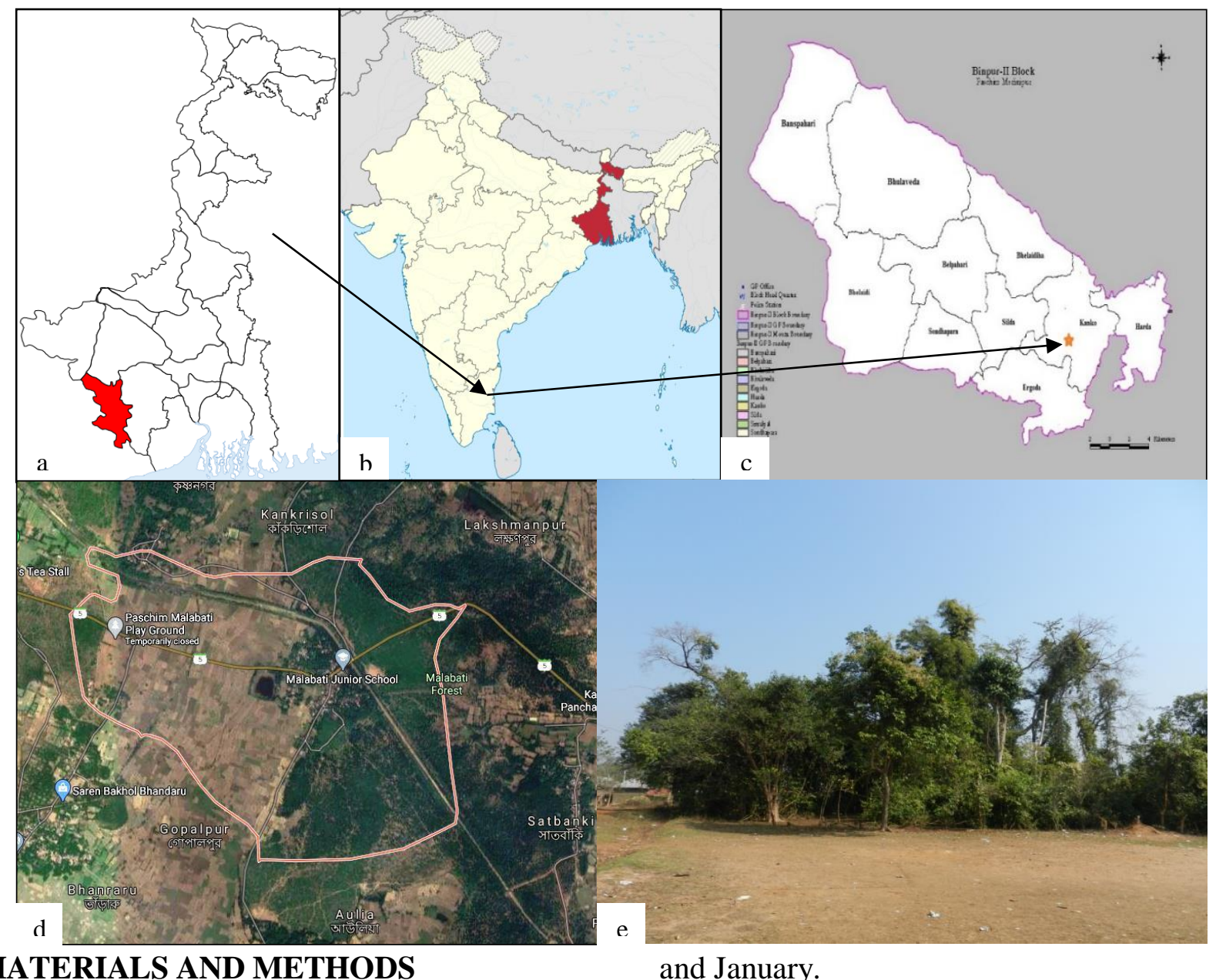

Fig. 1. Location of the study area: a. India; b. Jhargram district in West Bengal; c. Binpur II block showing Maa Mongalmoyee Than (MMT); d. Google earth image of MMT; e. MMT sacred grove.

Fig. 1 shows the Chota Nagpur Plateau slowly sloping downwards, and forming an undulating region of infertile rocks/soil laterite. Based on the 2011 census, the district of 
Jhargram covers an area of $3037.64 \mathrm{~km}^{2}$ and has a population of $1,136,548$. Furthermore, $96.52 \%$ of this population was rural, and only $3.48 \%$ were urban, while $20.11 \%$ and $29.37 \%$ belonged to the scheduled castes and tribes, respectively (WBPSPM, 2011).

This 2-acre grove represents an isolated forest patch in near-climax stage and is surrounded by crop fields and forest land. The devotees collect money and other utensils from around the region and neighboring areas, to celebrate worship. Meanwhile, livestock, including goats and chickens, are sacrificed on 4 Magh $\left(18 / 19^{\text {th }}\right.$ of January) to satisfy the goddess, and this is a deep-rooted custom. Also, an iconic idol of Goddess Mongolmoyee was worshipped within the sacred grove and by the local tribal people, while maintenance and managerial activities are carried out by the local communities.

Field survey and data collection. The study area was thoroughly investigated during different seasons, between 2012 and 2018. This presented a prospect of the flora composition and field interpretation during the entire flowering and fruiting of the maximum species quantity. Subsequently, phytosociological data were collected by laying $20 \times 20 \mathrm{~m}$ quadrates for tree species, $5 \times 5 \mathrm{~m}$ for shrubs as well lianas, and $1 \times 1 \mathrm{~m}$ for herbs and grasses. A brief floristic survey was carried out through a "spot identification" basis, and the specimens were then processed, preserved, poisoned and mounted on sheets of herbarium (Jain \& Rao, 2016). In addition, photographs of some common, locally uncommon, endemic and valuable plant species within the sacred grove were taken. The herbarium sheets were then described by matching properly annotated materials available at the Herbarium Section of Vidyasagar University as well as the Botanical Survey of India. Numerous related catalogues (Anderson, 1862), regional flora (Hooker, 1875; Prain, 1903; Haines, 1926), monographs (Mitra, 1958), revision works (Datta \& Majumdar, 1966) and other articles (Paria, 2005; Paria, 2010), were also consulted for identification purposes, while the scientific names were checked using the World Checklist of Vascular Plant (WCVP, 2020) website and confirmed after being deemed acceptable.

Analysis of vegetation. The floristic list is taxonomically arranged based on clade, order, and family, according to Angiosperm Phylogeny Group IV classification (Chase et al., 2016). Table 1 shows inferred habit, lifespan, flowering and fruiting time, Raunkiaer's life-form of each species with sub-type, leaf size, IUCN status and distribution within the grove. The resulting biological spectrum was then compared to the standard Raunkiaer spectrum, to determine the grove's phytoclimate (Raunkiaer, 1934; MullerDombois \& Ellenberg, 1974). Also, leaf size information was used to assess the plant physiological systems and identify plant associations, while the Raunkiaer (1934) diagram was used to quickly estimate the leaves in the field. Subsequently, a list of invasive alien species was prepared, as described by Reddy (2008).

Table 1. List of angiosperm plants in Maa Mongalmoyee Than (MMT) sacred grove.

Name of the Species $\quad$ :

MESANGIOSPERMS

MAGNOLIIDS

Piperales Bercht. \& J. Presl

Piperaceae Giseke

Peperomia pellucida (L.) Kunth

Aristolochiaceae Juss.

Aristolochia indica L.

Magnoliales Juss. ex Bercht. \& J. Presl

H A Nov.-Mar. Th $\mathrm{Na}$ NE

Annonaceae Juss. 
Artabotrys hexapetalus (L.f.) Bhandari

C P Apr.-Oct.

$\mathrm{Ph}$

$\mathrm{N}$

$\mathrm{Me} \quad \mathrm{NE}$

\section{INDEPENDENT LINEAGE: UNPLACED TO MORE INCLUSIVE CLADE}

\section{MONOCOTS}

Alismatales R.Br. ex Bercht. \& J. Presl

Araceae Juss.

Pothos scandens L.

Scindapsus officinalis (Roxb.) Schott

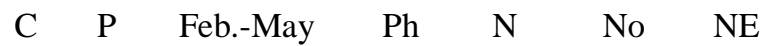

Dioscoreales Mart.

Dioscoreaceae R. Br.

Dioscorea bulbifera $\mathrm{L}$.

Asparagales Link

Orchidaceae Juss.

Habenaria commelinifolia (Roxb.) Wall. ex Lindl.

$\begin{array}{llllll}\mathrm{C} & \mathrm{P} & \mathrm{Ph} & \mathrm{N} & \mathrm{Mg} & \mathrm{NE}\end{array}$

Vanda tessellata (Roxb.) Hook. ex G. Don

Hypoxidaceae R.Br.

Curculigo capitulata (Lour.) Kuntze

Arecales Bromhead

Arecaceae Bercht. \& J. Presl

Phoenix acaulis Roxb.

Phoenix sylvestris (L.) Roxb.

Poales Small

Cyperaceae Juss.

Cyperus mindorensis (Steud.) Huygh

Cyperus distans L.f.

Cyperus rotundus L.

Fimbristylis aestivalis (Retz.) Vahl

Fimbristylis dichotoma (L.) Vahl

Fimbristylis eragrostis (Nees) Hance

Schoenoplectiella roylei (Nees) Lye

Scleria annularis Steud.

Poaceae Barnhart

Apluda mutica L.

Aristida setacea Retz.

Bothriochloa pertusa (L.) A. Camus

C P Aug.-Dec. $\mathrm{Cr}$

Ma NE

Cenchrus ciliaris L.

Chloris barbata $\mathrm{Sw}$.

Chrysopogon aciculatus (Retz.) Trin.

Cynodon dactylon (L.) Pers.

Dichanthium foveolatum (Delile) Roberty

Digitaria sanguinalis (L.) Scop.

Eleusine indica (L.) Gaertn.

Eragrostis cilianensis (All.) Vignolo ex Janch.

$\mathrm{H} \quad \mathrm{P}$ Aug.-Oct. $\mathrm{Cr} \quad \mathrm{Na}$ NE

H P Apr.-Jul. Ph N No

H P Jun.-Sep. $\quad$ Cr $\quad$ Me NE

Eragrostis unioloides (Retz.) Nees ex Steud.

S P Feb.-Jun. $\mathrm{Ch} \quad \mathrm{Me}$ NE

T P Feb.-Jun. Ph $\quad$ M $\quad$ Me NE

Heteropogon contortus (L.) P. Beauv. ex Roem. \& Schult.

Imperata cylindrica (L.) P.Beauv.

H P Nov.-Mar. He

$\mathrm{Na} \quad \mathrm{LC}$

$\mathrm{H} \quad \mathrm{P}$ Jul.-Sep. He Le LC

H P Sep.-Dec. He Le LC

$\mathrm{H} \quad \mathrm{P}$ Feb.-May He Le NE

$\mathrm{H} \quad \mathrm{P}$ Aug.-Oct. He Le LC

$\mathrm{H} \quad \mathrm{P}$ May-Aug. He Le NE

H P Feb.-Aug. He $\quad$ Le LC

$\begin{array}{lllll}\mathrm{H} & \mathrm{P} & \text { Feb.-Sep. } \mathrm{He} & \mathrm{Mi} & \mathrm{NE}\end{array}$

Oplismenus compositus (L.) P. Beauv.

Paspalum scrobiculatum $\mathrm{L}$.

Perotis indica (L.) Kuntze

Rottboellia cochinchinensis (Lour.) Clayton

Sacciolepis myosuroides (R.Br.) Chase ex E.G.Camus \&

A.Camus

Setaria flavida (Retz.) Veldkamp

Sporobolus diandrus (Retz.) P. Beauv.

Tragus mongolorum Ohwi

P Sep.-Nov. He

$\mathrm{Na} \quad \mathrm{NE}$

$\mathrm{H} \quad \mathrm{P}$ Aug.-Dec. He Le NE

$\mathrm{H}$ A Jan.-May $\mathrm{He} \quad \mathrm{Na}$ NE

$\mathrm{H} \quad \mathrm{P}$ Jun.-Oct. $\mathrm{He} \quad \mathrm{Na}$ LC

$\mathrm{H} \quad \mathrm{P}$ Aug.-Nov. He Le NE

$\mathrm{H} \quad \mathrm{P}$ Jun.-Oct. He Le NE

$\mathrm{H} \quad \mathrm{P}$ All $\mathrm{He} \quad \mathrm{Le}$ NE

$\mathrm{H} \quad \mathrm{P}$ All $\mathrm{He} \quad \mathrm{Le}$ NE

$\mathrm{H} \quad \mathrm{P}$ Mar.-Jun. $\mathrm{He}$ Le NE

$\mathrm{H} \quad \mathrm{P}$ Aug.-Nov. He Le LC

$\mathrm{H}$ P Jul.-Nov. He Le NE

$\mathrm{H} \quad \mathrm{P}$ Oct.-Jan He Le LC

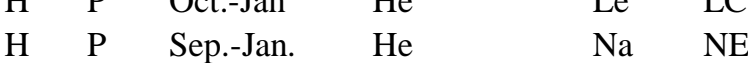

$\mathrm{H} \quad \mathrm{P}$ Oct.-Dec. $\mathrm{He} \quad \mathrm{Na}$ LC

$\mathrm{H} \quad \mathrm{P}$ Sep.-Dec. $\mathrm{He} \quad \mathrm{Mi}$ NE

$\mathrm{H}$ A Aug.-Nov. $\mathrm{He} \quad \mathrm{Na}$ LC

$\mathrm{H}$ P Jul.-Nov. He Le NE

$\mathrm{H} \quad \mathrm{P}$ Aug.-Oct. $\mathrm{He} \quad \mathrm{Mi}$ NE

$\mathrm{H} \quad \mathrm{P}$ Sep.-Dec. $\mathrm{He} \quad \mathrm{Na}$ LC

\section{EUDICOTS}

$\mathrm{H} \quad \mathrm{P}$ Aug.-Nov. He

$\mathrm{Na} \quad \mathrm{NE}$

$\mathrm{H} \quad \mathrm{P}$ Nov.-Jun. $\mathrm{He} \quad \mathrm{Na}$ NE

$\mathrm{H} \quad \mathrm{P}$ Aug.-Oct. $\mathrm{He}$ Le NE 
Ranunculales Juss. ex Bercht. \& J. Presl

Papaveraceae Juss.

Argemone mexicana L.

Menispermaceae Juss.

Cissampelos pareira $\mathrm{L}$.

Cocculus hirsutus (L.) W. Theob.

Stephania japonica (Thunb.) Miers

Tiliacora acuminata (Lam.) Miers

Tinospora sinensis (Lour.) Merr.

CORE EUDICOTS

\section{SUPERROSIDS}

Saxifragales Bercht. \& J. Presl

Crassulaceae J.St.-Hil.

Kalanchoe pinnata (Lam.) Pers.

\section{ROSIDS}

H P Mar.-Jun. Ch

$\mathrm{Ma} \quad \mathrm{NE}$

Vitales Juss. ex Bercht. \& J. Presl

Vitaceae Juss.

Causonis trifolia (L.) Mabb. \& J.Wen

Cayratia pedata (Lam.) Gagnep.

Cissus quinquangularis Chiov.

H A Dec.-Apr Th

$\mathrm{Ma} \quad \mathrm{NE}$

Fabales Bromhead

Fabaceae Lindl.

Abrus precatorius L.

Bauhinia acuminata $\mathrm{L}$.

Brachypterum scandens (Roxb.) Miq.

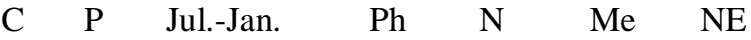

C P Aug.-Nov. Ph N No NE

C P Jul.-Dec. Ph $\mathrm{N}$ Me NE

C $\quad \mathrm{P}$ Nov.-May $\mathrm{Ph} \quad \mathrm{N} \quad \mathrm{Me}$ NE

C $\mathrm{P}$ Feb.-Jun. $\mathrm{Ph} \quad \mathrm{N} \quad \mathrm{Me}$ NE

Cajanus scarabaeoides (L.) Thouars

Cassia fistula L.

Chamaecrista absus (L.) H. S. Irwin \& Barneby

Crotalaria prostrata Rottler ex Willd.

Dicliptera bupleuroides Nees

Erythrina variegata $\mathrm{L}$.

Flemingia chappar Buch.-Ham. ex Benth.

Gliricidia sepium (Jacq.) Steud.

Grona triflora (L.) H.Ohashi \& K.Ohashi

Guilandina bonduc L.

Indigofera linnaei Ali

Mimosa pudica L.

Mimosa rubicaulis Lam.

Peltophorum pterocarpum (DC.) Backer ex K. Heyne

C $\quad \mathrm{P}$ Aug.-Dec. $\mathrm{Ph}$

C P Aug.-Feb. Ph

C P Aug.-Dec. Ph

$\begin{array}{lll}\mathrm{N} & \mathrm{No} & \mathrm{NE} \\ \mathrm{N} & \mathrm{Me} & \mathrm{VU} \\ \mathrm{N} & \mathrm{No} & \mathrm{NE}\end{array}$

Phanera vahlii (Wight \& Arn.) Benth.

C $\mathrm{P}$ Aug.-Mar. $\mathrm{Ph} \quad \mathrm{N} \quad \mathrm{Na} \quad \mathrm{NE}$

Pleurolobus gangeticus (L.) J.St.-Hil. ex H.Ohashi \&

K.Ohashi

Senna obtusifolia (L.) H. S. Irwin \& Barneby

Senna sophera (L.) Roxb.

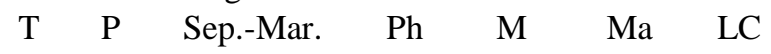

C P Jul.-Jan. $\mathrm{Ph} \quad \mathrm{N} \quad \mathrm{Na}$ NE

C A Sep.-Feb. Ph $\mathrm{N} \quad \mathrm{Mi}$ LC

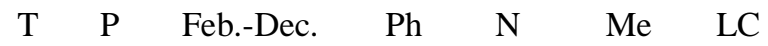

$\mathrm{H}$ A Aug.-Dec. Th $\mathrm{Mi}$ LC

$\mathrm{H}$ A Aug.-Jan. Th No NE

$\mathrm{H}$ A Jun.-Oct. Th No NE

T P Feb.-Jul. $\mathrm{Ph}$ M $\mathrm{Ma}$ LC

$\mathrm{S} \quad \mathrm{P}$ Nov.-Feb. $\mathrm{Ch} \quad \mathrm{Me}$ NE

T P Feb.-May Ph N $\quad$ Me LC

$\mathrm{H} \quad \mathrm{P}$ Jul.-Feb. Th $\mathrm{Me}$ NE

C P Aug.-Apr. Ph N $\mathrm{Mi}$ LC

$\mathrm{H}$ B Aug.-Nov. Th $\mathrm{Mi}$ NE

$\mathrm{H} \quad \mathrm{P}$ Jul.-Nov. Th $\mathrm{Na}$ LC

$\mathrm{S} \quad \mathrm{P}$ Jul.-Nov. $\mathrm{Ch}$ Me NE

Senna tora (L.) Roxb.

Smithia sensitiva Aiton

Tephrosia purpurea (L.) Pers.

Teramnus labialis (L. f.) Spreng.

Vachellia nilotica (L.) P.J.H.Hurter \& Mabb.

Zornia gibbosa Span.

Polygalaceae Hoffmanns. \& Link

Polygala arvensis Willd.

Rosales Bercht. \& J. Presl

T P Mar.-Jan. Ph

$\mathrm{MM} \quad \mathrm{Mi} \quad \mathrm{NE}$

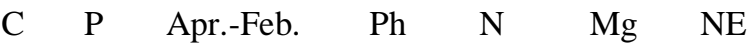

Rhamnaceae Juss.

Gouania tiliifolia Lam.

$\begin{array}{lllllll}\text { H } & \text { A } & \text { Mar.-Oct. } & \text { Th } & & \mathrm{Mi} & \text { LC } \\ \text { S } & \text { A } & \text { Apr.-Sep. } & \text { Ch } & & \text { No } & \text { NE } \\ \text { H } & \text { A } & \text { Sep.-Dec. } & \text { Th } & & \mathrm{Mi} & \text { NE } \\ \text { H } & \text { A } & \text { Oct.-Jan. } & \text { Th } & & \mathrm{Na} & \text { LC } \\ \text { H } & \text { P } & \text { Sep.-Dec. } & \text { Th } & & \mathrm{Na} & \text { LC } \\ \text { C } & \text { A } & \text { Aug.-Dec. } & \text { Ph } & \text { N } & \mathrm{Mi} & \text { NE } \\ \text { T } & \text { P } & \text { Jun.-Sep. } & \text { Ph } & \text { M } & \text { Na } & \text { LC } \\ \text { H } & \text { A } & \text { Aug.-Nov. } & \text { Th } & & \mathrm{Na} & \text { NE } \\ & & & & & & \\ \text { H } & \text { A } & \text { Jul.-Dec. } & \text { Th } & & \mathrm{Me} & \text { NE } \\ & & & & & & \\ & & & & & & \\ \text { C } & \text { P } & \text { Apr.-Oc. } & \text { Ph } & \text { N } & \mathrm{Me} & \text { NE }\end{array}$


Ventilago denticulata Willd.

Ziziphus oenopolia (L.) Mill.

Cannabaceae Martinov

Cannabis sativa $\mathrm{L}$.

Moraceae Gaudich.

Streblus asper Lour.

Cucurbitales Juss. ex Bercht. \& J. Presl

Cucurbitaceae Juss.

Cayaponia laciniosa (L.) C. Jeffrey

Coccinia grandis (L.) Voigt

Diplocyclos palmatus (L.) C. Jeffrey

Solena amplexicaulis (Lam.) Gandhi

Trichosanthes cucumerina $\mathrm{L}$.

Trichosanthes tricuspidata Lour.

Celastrales Link

Celastraceae R. Br.

Celastrus paniculatus Willd.

Oxalidales Bercht. \& J. Presl

Oxalidaceae R. Br.

Oxalis corniculata L.

Malpighiales Juss. ex Bercht. \& J. Presl

Violaceae Batsch

Afrohybanthus enneaspermus (L.) Flicker

Salicaceae Mirb.

Flacourtia indica (Burm. f.) Merr.

Euphorbiaceae Juss.

Acalypha indica $\mathrm{L}$.

Baliospermum solanifolium (Burm.) Suresh

Chrozophora plicata (Vahl) A. Juss. ex Spreng.

Croton bonplandianus Baill.

Euphorbia hirta L.

Euphorbia thymifolia L.

Jatropha gossypiifolia L.

Tragia involucrata $\mathrm{L}$.

Phyllanthaceae Martinov

Breynia vitis-idaea (Burm.f.) C. E. C. Fisch.

Phyllanthus lanceolarius (Roxb.) Müll.Arg.

Myrtales Juss. ex Bercht. \& J. Presl

Combretaceae R. Br.

Combretum decandrum Jacq.

Lythraceae J. St.-Hil.

Woodfordia fruticosa (L.) Kurz

Sapindales Juss. ex Bercht. \& J. Presl

Sapindaceae Juss.

Allophylus serratus (Roxb.) Kurz

Cardiospermum halicacabum L.

Rutaceae Juss.

Aegle marmelos (L.) Corrêa

Meliaceae Juss.

Azadirachta indica A. Juss.

Malvales Juss. ex Bercht. \& J. Presl

Malvaceae Juss.

Abelmoschus crinitus Wall.

Abroma augusta (L.) L. f.

Abutilon indicum (L.) Sweet

$\begin{array}{lllllll}\text { C } & \text { P } & \text { Sep.-Jun. } & \text { Ph } & \text { N } & \text { Me } & \text { NE } \\ \text { C } & \text { P } & \text { Nov.-Mar. } & \text { Ph } & \text { N } & \text { No } & \text { LC } \\ & & & & & & \\ \text { S } & \text { A } & \text { Oct.-Feb. } & \text { Ch } & & \text { Mg } & \text { NE } \\ & & & & & & \\ \text { T } & \text { P } & \text { Feb.-Jun. } & \text { Ph } & \text { N } & \text { Mi } & \text { LC }\end{array}$

C $\quad \mathrm{P}$ Mar.-Dec. $\mathrm{Ph} \quad \mathrm{N} \quad \mathrm{Me}$ NE

C $\mathrm{P}$ Mar.-Dec. $\mathrm{Ph} \quad \mathrm{N} \quad \mathrm{Me}$ NE

C $\mathrm{P}$ Aug.-Oct. $\mathrm{Ph}$ N $\mathrm{Me}$ NE

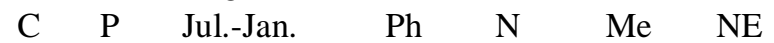

C $\mathrm{P}$ Aug.-Dec. $\mathrm{Ph} \quad \mathrm{N} \quad \mathrm{Me}$ NE

C A Apr.-Sep. Ph N $\mathrm{Ne}$ NE

C $\quad \mathrm{P}$ Apr.- Dec. $\mathrm{Ph} \quad \mathrm{N} \quad \mathrm{Me} \quad \mathrm{NE}$

$\begin{array}{lllll}\mathrm{H} & \mathrm{A} & \mathrm{All} & \mathrm{Th} & \mathrm{Na}\end{array}$

$\begin{array}{lllll}\mathrm{H} & \mathrm{P} & \text { Jul.-Nov. } & \mathrm{Th} & \mathrm{Na}\end{array}$

S P Sep.-May Ch No

H A All Th No NE

S A Aug.-Mar. Th Me LC

$\mathrm{H}$ A Feb.-Apr. Th No NE

$\mathrm{H} \quad \mathrm{P}$ All $\quad$ Th $\quad$ No NE

$\mathrm{H}$ A Feb.-Dec. Th $\mathrm{Na}$ NE

$\begin{array}{lllll}\mathrm{H} & \mathrm{P} & \mathrm{All} & \mathrm{Th} & \mathrm{N}\end{array}$

S P Apr.-Aug. $\mathrm{Ch}$ Ma LC

C P Mar.-Jan. Ph N $\mathrm{Me}$ NE

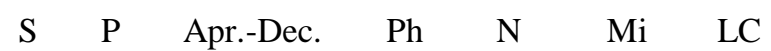

S A Mar.-Dec. Th Me LC

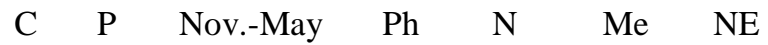

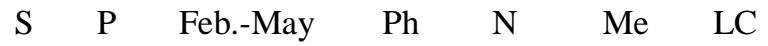

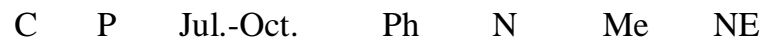

C A Jul.-Dec. Ph N No LC

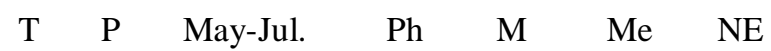

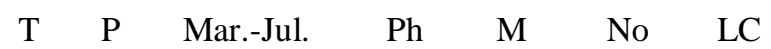

S A Mar.-Sep. Ch Ma LC

S P Jan.-Mar. Ph N Ma NE

$\mathrm{S}$ A Jun.- Dec. $\mathrm{Ch}$ Ma NE 
Azanza lampas (Cav.) Alef.

Grewia asiatica $\mathrm{L}$.

Corchorus aestuans L.

Helicteres isora $\mathrm{L}$.

Malvastrum coromandelianum (L.) Garcke

Melochia corchorifolia L.

Sida cordata (Burm. f.) Borss. Waalk.

Sida cordifolia L.

Sida rhombifolia L.

Urena lobata $\mathrm{L}$.

Capparaceae Juss.

Capparis zeylanica L.

Cleomaceae Bercht. \& J. Presl

Cleome viscosa $\mathrm{L}$.

\section{SUPERASTERIDS}

Santalales R.Br. ex Bercht. \& J. Presl

Santalaceae R.Br.

Viscum cruciatum Sieber ex Boiss.

Viscum multinerve (Hayata) Hayata

Loranthaceae Juss.

Dendrophthoe falcata (L. f.) Ettingsh.

Scurrula cordifolia (Wall.) G.Don

Caryophyllales Juss. ex Bercht. \& J. Presl

Plumbaginaceae Juss.

Plumbago zeylanica L.

Polygonaceae Juss.

Antigonon leptopus Hook. \& Arn.

Amaranthaceae Juss.

Achyranthes aspera L.

Alternanthera sessilis (L.) R.Br. ex DC.

Amaranthus spinosus $\mathrm{L}$.

Amaranthus viridis L.

Dysphania ambrosioides (L.) Mosyakin \& Clemants

Gomphrena celosioides Mart.

Ouret lanata (L.) Kuntze

Pupalia lappacea (L.) Juss.

Aizoaceae Martinov

Trianthema portulacastrum L.

Nyctaginaceae Juss.

Boerhavia erecta L.

Molluginaceae Bartl.

Trigastrotheca pentaphylla (L.) Thulin

Cactaceae Juss.

Cereus hexagonus (L.) Mill.

\section{ASTERIDS}

Cornales Link

Cornaceae Bercht. \& J. Presl

Alangium salviifolium (L. f.) Wangerin

Ericales Bercht. \& J. Presl

Sapotaceae Juss.

Madhuca longifolia var. latifolia (Roxb.) A. Chev.

Ebenaceae Gurke

Diospyros melanoxylon Roxb.

Diospyros montana Roxb.

Gentianales Juss. ex Bercht. \& J. Presl

$\begin{array}{lllllll}\text { S } & \text { A } & \text { Sep.-Dec. } & \text { Ch } & & \text { Ma } & \text { NE } \\ \text { T } & \text { P } & \text { Jun.-Aug. } & \text { Ph } & \text { N } & \text { Ma } & \text { LC } \\ \text { H } & \text { A } & \text { Jul.-Nov. } & \text { Th } & & \text { Me } & \text { NE } \\ \text { S } & \text { P } & \text { Sep.- Feb. } & \text { Ph } & \text { N } & \text { Me } & \text { NE } \\ \text { H } & \text { A } & \text { Jul.-Nov. } & \text { Th } & & \text { No } & \text { NE } \\ \text { H } & \text { A } & \text { May-Jun. } & \text { Th } & & \text { No } & \text { LC } \\ \text { H } & \text { A } & \text { Aug.-Feb. } & \text { Th } & & \text { No } & \text { NE } \\ \text { S } & \text { A } & \text { Aug.-Dec. } & \text { Th } & & \text { No } & \text { NE } \\ \text { H } & \text { P } & \text { Sep.-Dec. } & \text { Th } & & \text { No } & \text { NE } \\ \text { S } & \text { A } & \text { Sep.-Dec. } & \text { Ch } & & \text { No } & \text { LC } \\ & & & & & & \\ \text { C } & \text { P } & \text { Mar.-Oct. } & \text { Ph } & \text { M } & \text { No } & \text { NE } \\ & & & & & & \\ \text { H } & \text { A } & \text { Sep.-Apr. } & \text { Th } & & \text { No } & \text { NE }\end{array}$

S P Jan.-Jun. $\mathrm{Ph} \quad \mathrm{N}$ Le NE

S P Mar.-Jul. Ph N Le NE

S A Nov.-Mar. Ph N No NE

S A Jul.-Nov. Ph N No NE

H A Sep.-Feb. Th $\quad$ Mi NE

C A Aug.-Jan. $\mathrm{Ph} \quad \mathrm{N} \quad \mathrm{Me}$ NE

H A Sep.-Feb. Th

$\mathrm{H}$ A Jul.-Feb. Th $\mathrm{Mi}$ LC

$\mathrm{H}$ A All $\quad$ Th $\quad \mathrm{Na}$ NE

$\mathrm{H}$ A All $\quad \mathrm{Th} \quad \mathrm{Na}$ NE

$\mathrm{H}$ A Apr.-Jul. Th $\mathrm{Mi}$ NE

$\mathrm{H}$ A Apr.-Aug. Th No NE

H A Nov.-Jan. Th Le NE

H P Sep.-Jan. Th NC

H A Apr.-Oct. Th

H A Jul.-Sep. Th $\quad$ Mi NE

H A Aug.-Nov. Th $\quad$ Na NE

S P Jun.-Jul. $\quad$ Ch $\quad$ Le LC

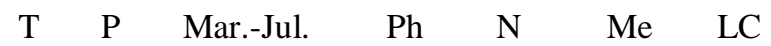

T $\quad$ P Mar.-Jul. $\quad \mathrm{Ph} \quad \mathrm{MM}$ Ma NE

T $\quad$ P Apr.-Jul. $\quad$ Ph $\quad$ MM Ma NE

T P Apr.-Jan. Ph MM Ma NE 
Rubiaceae Juss.

Adina cordifolia (Roxb.) Brandis

Cissus quinquangularis Chiov.

Dentella repens (L.) J. R. Forst. \& G. Forst.

Meyna laxiflora Robyns

Morinda citrifolia L.

Oldenlandia corymbosa L.

Paederia foetida $\mathrm{L}$.

Spermacoce lasiocarpa $\mathrm{R}$. Br. ex Wall.

Gentianaceae Juss.

Canscora diffusa (Vahl) R. Br. ex Roem. \& Schult.

Apocynaceae Juss.

Aganosma heynei (Spreng.) I. M. Turner

Calotropis gigantea (L.) W.T.Aiton

Cryptolepis dubia (Burm. f.) M. R. Almeida

Gymnema sylvestre (Retz.) R. Br. ex Sm.

Hemidesmus indicus (L.) R.Br.

Ichnocarpus frutescens (L.) W.T. Aiton

Pergularia daemia (Forssk.) Chiov.

Rauvolfia tetraphylla L.

Telosma pallida (Roxb.) Craib

Vallaris solanacea (Roth) Kuntze

Solanales Juss. ex Bercht. \& J. Presl

Convolvulaceae Juss.

Argyreia nervosa (Burm. f.) Bojer

Cuscuta reflexa Roxb.

Erycibe paniculata Roxb.

Evolvulus alsinoides (L.) L.

Evolvulus nummularius (L.) L.

Ipomoea mauritiana Jacq.

Merremia emarginata (Burm. f.) Hallier f.

Rivea ornata (Roxb.) Choisy

Solanaceae Juss.

Datura stramonium L.

Nicotiana plumbaginifolia Viv.

Physalis angulata L.

Solanum americanum Mill.

Solanum torvum $\mathrm{Sw}$.

Solanum virginianum $\mathrm{L}$.

Lamiales Bromhead

Oleaceae Hoffmanns. \& Link

Jasminum multiflorum (Burm. f.) Andrews

Plantaginaceae Juss.

Scoparia dulcis L.

Veronica anagallis-aquatica L.

Scrophulariaceae Juss.

Buddleja asiatica Lour.

Acanthaceae Juss.

Andrographis echioides (L.) Nees

Dicliptera paniculata (Forssk.) I. Darbysh.

Hemigraphis hirta (Vahl) T. Anderson

Justicia adhatoda L.

Justicia gendarussa Burm.f.

Rostellularia procumbens (L.) Nees

Ruellia prostrata Poir.

\begin{tabular}{|c|c|c|c|c|c|}
\hline $\mathrm{T}$ & $\mathrm{P}$ & Jun.-Dec. & $\mathrm{Ph}$ & MM & $\mathrm{Me}$ \\
\hline $\mathrm{C}$ & $\mathrm{P}$ & Feb.-Apr. & $\mathrm{Ph}$ & $\mathrm{N}$ & $\mathrm{Mi}$ \\
\hline $\mathrm{H}$ & A & Feb.-May & $\mathrm{Th}$ & & $\mathrm{Na}$ \\
\hline S & $\mathrm{P}$ & Mar.-Jun. & $\mathrm{Ch}$ & & $\mathrm{Me}$ \\
\hline $\mathrm{T}$ & $\mathrm{P}$ & Feb.-May & $\mathrm{Ph}$ & $\mathrm{N}$ & $\mathrm{Ma}$ \\
\hline $\mathrm{H}$ & A & Aug.-Feb. & Th & & Le \\
\hline $\mathrm{C}$ & $\mathrm{P}$ & Sep.-Dec. & $\mathrm{Ch}$ & & No \\
\hline $\mathrm{H}$ & A & All & Th & & $\mathrm{Na}$ \\
\hline $\mathrm{H}$ & A & Oct.-Jan. & Th & & Le \\
\hline $\mathrm{C}$ & $\mathrm{P}$ & Jul.-Nov. & $\mathrm{Ph}$ & $\mathrm{N}$ & No \\
\hline S & $\mathrm{P}$ & Mar.-Feb. & $\mathrm{Ch}$ & & $\mathrm{Ma}$ \\
\hline $\mathrm{C}$ & $\mathrm{P}$ & Apr.-Mar. & $\mathrm{Ph}$ & $\mathrm{N}$ & No \\
\hline $\mathrm{C}$ & $\mathrm{P}$ & Apr.-Mar. & $\mathrm{Ph}$ & $\mathrm{N}$ & Mi \\
\hline $\mathrm{C}$ & $\mathrm{P}$ & Aug.-Jan. & $\mathrm{Ph}$ & $\mathrm{N}$ & $\mathrm{Mi}$ \\
\hline $\mathrm{C}$ & $\mathrm{P}$ & Sep.-Mar. & $\mathrm{Ph}$ & $\mathrm{N}$ & No \\
\hline $\mathrm{C}$ & $\mathrm{P}$ & Sep.-Jan. & $\mathrm{Ph}$ & $\mathrm{N}$ & $\mathrm{Me}$ \\
\hline $\mathrm{S}$ & $\mathrm{P}$ & Feb.-Dec. & $\mathrm{Ch}$ & & No \\
\hline $\mathrm{C}$ & $\mathrm{P}$ & Sep.-Feb. & $\mathrm{Ph}$ & $\mathrm{N}$ & $\mathrm{Me}$ \\
\hline $\mathrm{C}$ & $\mathrm{P}$ & Apr.-Jan. & $\mathrm{Ph}$ & $\mathrm{N}$ & $\mathrm{Me}$ \\
\hline
\end{tabular}

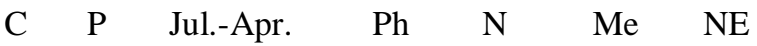

C P Oct.-Feb. Ph N Ap NE

$\mathrm{S}$ A Apr.-Jun. Ch No NE

$\mathrm{H}$ A Jul.-Feb. Th $\mathrm{Na}$ NE

$\mathrm{H}$ A All Th $\mathrm{Na}$ NE

C $\mathrm{P}$ Aug.-Dec. $\mathrm{Ph}$ N $\mathrm{Ma}$ NE

C $\mathrm{P}$ Dec.-Apr. Ph N No LC

C P Aug.-Oct. Ph N No NE

S P Jul.-Oct. Ch $\quad$ Ma NE

$\mathrm{H}$ A May-Sep. Th Ma NE

$\mathrm{S}$ A Aug.-Dec. $\mathrm{Ch}$ Ma LC

$\mathrm{H}$ A Oct.-Apr. Th $\mathrm{Mi}$ NE

S P Jul.-Mar. $\mathrm{Ch}$ Ma NE

$\mathrm{H}$ A Dec.-Jun. Th Ma NE

C $\mathrm{P}$ Dec.-Mar. $\mathrm{Ph} \quad \mathrm{N}$ No

H A May-Dec. Th $\mathrm{Mi}$ NE

$\mathrm{H}$ A Feb.-Jun. Th $\mathrm{Mi}$ LC

S P Jan.-Oct. Ch $\quad$ No LC

H A Jul.-Oct. Th No NE

$\mathrm{H}$ A Dec.-Feb. Th $\mathrm{Mi}$ NE

$\mathrm{H}$ A Aug.-Nov. Th $\mathrm{Mi}$ NE

S P Feb.-Apr. $\mathrm{Ch} \quad \mathrm{Me}$ NE

S P Feb.-Apr. Ch $\quad$ Me NE

$\mathrm{H} \quad \mathrm{P}$ Oct.-Jan. Th $\mathrm{Me}$ NE

$\mathrm{H}$ A May-Oct. Th Mi NE 


\begin{tabular}{|c|c|c|c|c|c|c|c|}
\hline Ruellia tuberosa $\mathrm{L}$. & $\mathrm{H}$ & A & Aug.-Nov. & $\mathrm{Th}$ & & $\mathrm{Mi}$ & $\mathrm{NE}$ \\
\hline Rungia pectinata (L.) Nees & $\mathrm{H}$ & A & All & $\mathrm{Th}$ & & $\mathrm{Mi}$ & $\mathrm{NE}$ \\
\hline \multicolumn{8}{|l|}{ Verbenaceae J. St. Hil. } \\
\hline Lantana camara $\mathrm{L}$. & $\mathrm{S}$ & $\mathrm{P}$ & Nov.-Feb. & $\mathrm{Ch}$ & & No & $\mathrm{NE}$ \\
\hline \multicolumn{8}{|l|}{ Lamiaceae Martinov } \\
\hline Anisomeles indica (L.) Kuntze & $\mathrm{H}$ & A & Sep.-Jan. & $\mathrm{Ch}$ & & No & $\mathrm{NE}$ \\
\hline Clerodendrum infortunatum $\mathrm{L}$. & $\mathrm{S}$ & $\mathrm{P}$ & Feb.-Jul. & $\mathrm{Ch}$ & & $\mathrm{Ma}$ & $\mathrm{NE}$ \\
\hline Leonotis nepetifolia (L.) R.Br. & $\mathrm{H}$ & A & Apr.-Jul. & Th & & $\mathrm{Me}$ & $\mathrm{NE}$ \\
\hline Mesosphaerum suaveolens (L.) Kuntze & $\mathrm{H}$ & A & Sep.-Jan. & $\mathrm{Ch}$ & & $\mathrm{Me}$ & $\mathrm{NE}$ \\
\hline Ocimum americanum $\mathrm{L}$. & $\mathrm{H}$ & $\mathrm{P}$ & All & $\mathrm{Ch}$ & & $\mathrm{Na}$ & $\mathrm{NE}$ \\
\hline Ocimum basilicum $\mathrm{L}$. & $\mathrm{H}$ & $\mathrm{P}$ & May-Jul. & $\mathrm{Ch}$ & & $\mathrm{Na}$ & $\mathrm{NE}$ \\
\hline Vitex glabrata $\mathrm{R} . \mathrm{Br}$. & $\mathrm{T}$ & $\mathrm{P}$ & Apr.-Sep. & $\mathrm{Ph}$ & $\mathrm{N}$ & $\mathrm{Me}$ & $\mathrm{LC}$ \\
\hline Volkameria inermis L. & $\mathrm{S}$ & $\mathrm{P}$ & Nov.-Jan. & $\mathrm{Ch}$ & & No & $\mathrm{NE}$ \\
\hline \multicolumn{8}{|l|}{ Orobanchaceae Vent. } \\
\hline Orobanche aegyptiaca Pers. & $\mathrm{H}$ & A & Sep.-Feb. & $\mathrm{Cr}$ & & Le & $\mathrm{NE}$ \\
\hline Striga angustifolia (D. Don) C. J. Saldanha & $\mathrm{H}$ & A & Jul.-Nov. & Th & & Le & $\mathrm{NE}$ \\
\hline \multicolumn{8}{|l|}{ Asterales Link } \\
\hline \multicolumn{8}{|l|}{ Asteraceae Bercht. \& J. Presl } \\
\hline Acanthospermum hispidum DC. & $\mathrm{H}$ & A & Nov.-Feb. & Th & & No & $\mathrm{NE}$ \\
\hline Ageratum conyzoides L. & $\mathrm{H}$ & A & Nov.-Mar. & Th & & $\mathrm{Mi}$ & $\mathrm{LC}$ \\
\hline Blainvillea acmella (L.) Philipson & $\mathrm{H}$ & A & Dec.-Mar. & Th & & No & $\mathrm{LC}$ \\
\hline Blumea lacera (Burm.f.) DC. & $\mathrm{H}$ & A & Aug.-Feb. & $\mathrm{Th}$ & & Mi & $\mathrm{LC}$ \\
\hline Chromolaena odorata (L.) R. M. King \& H. Rob. & $\mathrm{H}$ & A & Mar.-Sep. & $\mathrm{Ch}$ & & Mi & $\mathrm{NE}$ \\
\hline Cyanthillium cinereum (L.) H. Rob. & $\mathrm{H}$ & A & Aug.-Mar. & Th & & $\mathrm{Mi}$ & $\mathrm{NE}$ \\
\hline Elephantopus scaber L. & $\mathrm{H}$ & A & Sep.-Jan. & Th & & No & $\mathrm{NE}$ \\
\hline Gnaphalium polycaulon Pers. & $\mathrm{H}$ & A & Feb.-Apr. & Th & & Le & $\mathrm{LC}$ \\
\hline Grangea maderaspatana (L.) Poir. & $\mathrm{H}$ & A & Dec.-May & Th & & Le & $\mathrm{LC}$ \\
\hline Mikania micrantha Kunth & $\mathrm{C}$ & A & Sep.-Feb. & $\mathrm{Ph}$ & $\mathrm{N}$ & No & $\mathrm{NE}$ \\
\hline Sphaeranthus indicus L. & $\mathrm{H}$ & A & Nov.-Apr. & Th & & $\mathrm{Na}$ & $\mathrm{LC}$ \\
\hline Sphagneticola calendulacea (L.) Pruski & $\mathrm{H}$ & A & Apr.-Oct. & Th & & Mi & $\mathrm{NE}$ \\
\hline Xanthium strumarium L. & $\mathrm{H}$ & A & Sep.-Apr. & Th & & $\mathrm{Me}$ & $\mathrm{NE}$ \\
\hline
\end{tabular}

Notes: Abbreviation: Habit= C-Climber, H= Herb, S= Shrub, T= Tree; Life-Span: A= Annual, B= Biennial, P= Perennial; Raunkiaer's Life-form and Sub-type: $\mathrm{Ch}=$ Chamaephytes, $\mathrm{Cr}=$ Cryptophytes, $\mathrm{He}=$ Hemicryptophytes, $\mathrm{MM}=$ Megaphanerophytes, $\mathrm{M}=$ Mesophanerophyte, $\mathrm{N}=$ Nanophanerophytes, $\mathrm{Ph}=$ Phanerophytes, $\mathrm{Th}=$ Therophytes; Flowering and Fruiting time: Jan.= January, Feb.= February, Mar.= March, Apr.= April, Jun.= June, Jul.= July, Aug. $=$ August, Sep. $=$ September, Oct. $=$ October, Nov. $=$ November, Dec. $=$ December, All $=$ All season; Leaf spectra: Ap $=$ Aphyllous, Le $=$ Leptophyll, $\mathrm{Ma}=$ Macrophyll, $\mathrm{Me}=$ Mesophyll, Mg= Megaphyll, Mi= Microphyll, Na= Nanophyll, No= Notophyll; IUCN Red List status: LC= Least Concerned, $\mathrm{NE}=$ Not Evaluated, $\mathrm{VU}=$ Vulnerable

\section{RESULTS AND DISCUSSION}

Taxonomic composition. A total of 217 taxa (species and infraspecies) belonging to 196 genera, 59 families and 27 orders, were recorded. Based on this composition, the top two clades were Asterids and Rosids, while over $70 \%$ of the flora belong to Eudicot and Core Eudicot orders. According to Table 1 and Fig. 2, the most represented orders $(\geq 10$ species) are from Poales (30 or 13.82\%), Fabales (28 or $12.90 \%$ ), Lamiales (23 or
$10.60 \%$ ), Gentianales (19 or $8.76 \%$ ), Malvales (15 or $6.91 \%$ ), Caryophyllales (14 or $6.45 \%$ ), Solanales (14 or $6.45 \%$ ), Asterales (13 or $5.99 \%$ ) and Malpighiales 12 (5.53\%). A study by Gastauer et al., (2017) highlighted a similar distribution, while Gnanasekaran et al. (2012) reported similar studies in a sacred grove on angiosperms of Cuddalore district, Tamil Nadu, India. Pérez-Luque et al. (2014) has also illustrated similar types of order contribution. 


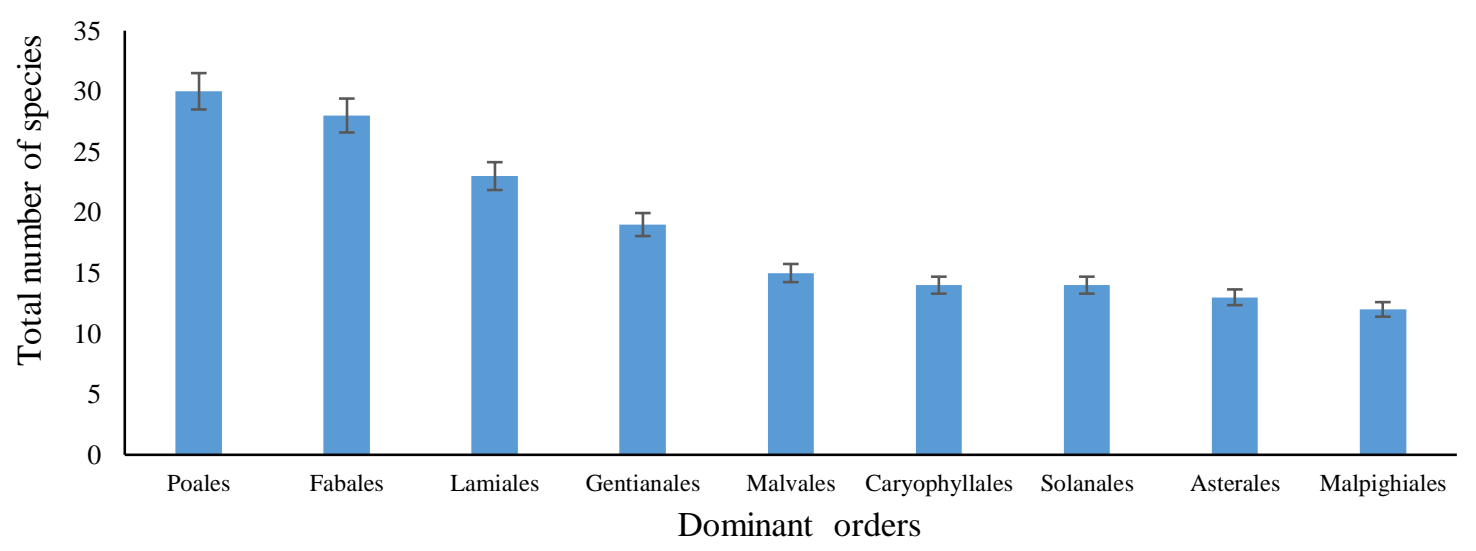

Fig. 2. Dominant orders ( $\geq 10$ species) in the Maa Mongalmoyee Than (MMT).

Fig. 2 shows the best-represented families (with $\geq 10$ species) are Fabaceae 27 (12.44\%), Poaceae 22 (10.14\%), Asteraceae 13 (5.99\%), Malvaceae $13(5.99 \%)$ and Apocynaceae 10 $(4.61 \%)$. Meanwhile, one family comprised 9 (4.15\%), another family covered $5(2.30 \%)$, six families contained $8(3.69 \%)$, two families contained $6(2.76 \%)$, two families covered 3 $(1.38 \%)$ and ten families comprised $2(0.92 \%)$ species, each. Also, 32 other families were found contain only a single species each. Numerous scholars (Ghildiyal et al., 2016; Sen $\&$ Bhakat, 2019) discovered the same type of dominant families, in sacred groves within India. These hegemony was also reported by many other authors (Badshah et al., 2016; Haq et al., 2018; Khan et al., 2018; Xu et al., 2018; Farooq et al., 2019; Asif et al., 2020). This is in accordance with the results, stating Fabaceae, Poaceae and Asteraceae, are the most represented, within the investigated area. Meanwhile, the most represented five genera are Cyperus, Fimbristylis, Senna, Sida and Solanum, comprising three species, each. Also, twelve genera, including Amaranthus, Diospyros, Eragrostis, Euphorbia, Evolvulus, Justicia, Mimosa, Ocimum, Phoenix, Ruellia, Trichosanthes and Viscum hold two species each. Table 1 shows the remaining 157 species, containing a single genus, each.

Species diversity in different growth form. This study showed the sacred grove harboured a total of 217 plant species (Table 2), [179 dicots $(82.49 \%)$ and 38 monocots (17.51\%)], belonging to196 genera [163 dicots $(83.16 \%)$ and 33 monocots $(16.84 \%)], 59$ families [52 dicots (88.14\%) and 7 monocots $(11.86 \%)$ ], and 27 orders [22 dicots $(81.48 \%)$ and 5 monocots $(18.52 \%)$ ].

Table 2. Total angiospermic taxa.

\begin{tabular}{lllllllll} 
Group & \multirow{2}{*}{ Orders } & Families & \multirow{2}{*}{ Genera } & $\begin{array}{l}\text { Species } \\
\text { Herbs }\end{array}$ & Shrubs & Trees & Climber & Total \\
\hline Dicots & 22 & 52 & 163 & 78 & 35 & 17 & 49 & 179 \\
Monocots & 5 & 7 & 33 & 33 & 1 & 1 & 3 & 38 \\
Total & 27 & 59 & 196 & 111 & 36 & 18 & 52 & 217 \\
\hline
\end{tabular}

Furthermore, 111(51.15\%) of the reported species were classified as herbs, 36 as shrubs (16.59\%), 18 as trees (8.29\%) and 52 as climbers $(23.97 \%)$. According to Table 1 and Table 2, herbs, shrubs, trees and climbers represented 78, 35, 17, 49 and 33, 1, 1, 3 species respectively, of the total $179(82.49 \%)$ dicots and monocots $38(17.51 \%)$ monocots. These denoted $35.94 \%, 16.13 \%, 7.83 \%, 22.58 \%$ and
$15.21 \%, 0.46 \%, 0.46 \%, 1.38 \%$, respectively, of the total species.

Life span. Table 1 shows the life cycle classification of flora in the sacred grove. Based on this classification, $86(39.63 \%)$ plants are annual (go through their life cycle in one growing season), $1(0.46 \%)$ plant is biennial (life cycle of two years) and 130 (59.91\%) plants are perennial (stay alive for more than 
two years), and able to survive most unfavorable conditions.

Life form and biological spectrum. The biological spectrum shows phanerophytes [76 (35.02\%)] are most frequent, followed by therophytes [73 (33.64\%)], chamaephytes [33 $(15.21 \%)$, hemicryptophytes [30 (13.82\%)] and cryptophytes [5 (2.30\%)]. Of the phanerophytes, nanophanerophytes [64 $(29.49 \%)$ are more common than mesophanerophytes $\left[\begin{array}{ll}7 & (3.23 \%)\end{array}\right]$ and megaphanerophytes [5(2.30\%)] (Table 3, Fig. $3)$.

Table 3. Biological spectrum (\% of all life forms) of study site and its comparison with Raunkiaer's normal spectrum.

\begin{tabular}{lllll}
\hline Life forms & $\begin{array}{l}\text { Total no. of } \\
\text { species }\end{array}$ & $\begin{array}{l}\text { Biological spectrum } \\
(\%) \text { of the study site }\end{array}$ & $\begin{array}{l}\text { Raunkiaer's normal } \\
\text { spectrum }(\%)\end{array}$ & $\begin{array}{l}\text { Deviation= (Raunkiaer's } \\
\text { normal spectrum- } \\
\text { Biological spectrum })\end{array}$ \\
\hline Phanerophytes & 76 & 35.02 & 46.00 & -10.98 \\
Megaphanerophytes & 5 & 2.30 & 3.00 & -0.7 \\
Mesophanerophytes & 7 & 3.23 & 28.00 & -24.77 \\
Nanophanerophytes & 64 & 29.49 & 15.00 & 14.49 \\
Chamaephytes & 33 & 15.21 & 9.00 & 6.21 \\
Hemicryptophytes & 30 & 13.82 & 26.00 & -12.18 \\
Cryptophytes & 5 & 2.30 & 6.00 & -3.7 \\
Therophytes & 73 & 33.64 & 13.00 & 20.64 \\
Total & $\mathbf{2 1 7}$ & 100 & $\mathbf{1 0 0}$ & \\
\hline
\end{tabular}

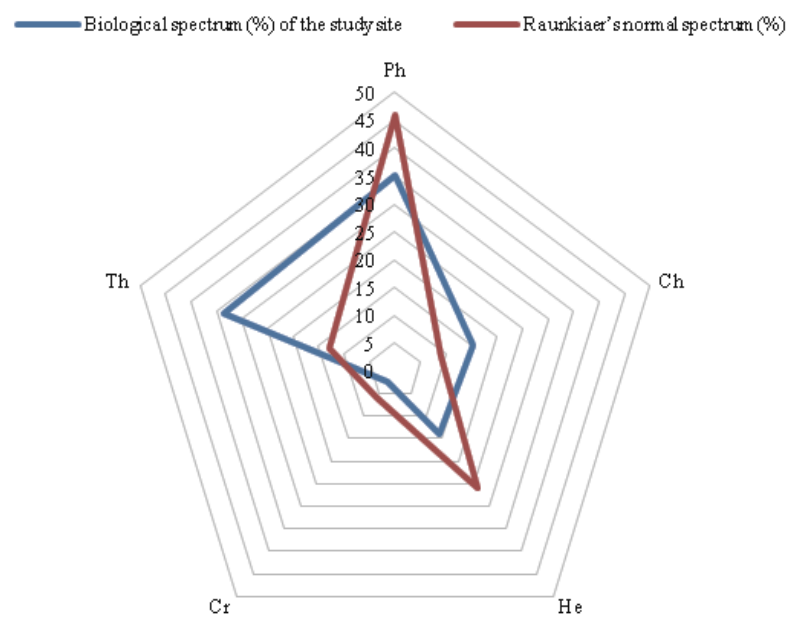

Fig. 3. Comparison of biological spectrum of MMT with Raunkiaer's normal spectra $(\mathrm{Ph}=$ Phanerophytes; $\mathrm{Ch}=$ Chamaephytes; $\mathrm{He}=$ Hemicryptophytes; $\mathrm{Cr}=$ Cryptophytes; $\mathrm{Th}=$ Therophytes).

Furthermore, the grove was found to constitute a higher percentage of therophytes (20.64\%) and cryptophytes (6.21\%), and a lower percentage of hemicryptophytes $(12.18 \%)$ and phanerophytes (10.98\%), compared to the normal spectrum exhibiting "thero-chamaephytic" phytoclimate. Meanwhile, of the phanerophytes, nanophanerophytes (14.49\%) are somewhat larger, while mesophanerophyte $(24.77 \%)$ and megaphanerophytes $(0.7 \%)$ are comparatively smaller than the normal spectrum (Table 3).
Therefore, the flora was compared to the standard range, developed by Raunkiaer for the flora world (1934), reflecting homogeneous climatic conditions (Sharma \& Raina 2010). The $\chi^{2}$ test results showed a significant difference $\left(\chi^{2}=47.663, \mathrm{df}=4, \mathrm{P}<0.0001\right)$ between the observed flora and Raunkiaer's normal spectrum. Table 3 shows this result is significant at $\mathrm{P}<0.05$, and regarded as extremely statistically significant (de Paula et $a l .$, 2017). The phanerophytes life form has the highest percentage, partly due to the local protection against certain taboos of the sacred grove.

According to Sharma \& Raina (2017), the highest percentage of therophytes within the area, is related to the subtropic character and often associated with various factors, including soil and climatic conditions, combined with grazing, lopping, felling, deforestation, introduction of annual weeds, and other anthropogenic activities. Thus, the present study indicates the flora are mostly subtropical, and with a higher percentage of therophytes as well as chamaephytes, compared to normal biological spectrum. Therophytes were also discovered to be strongly preferred in disturbed areas, and to be prevalent in regions having biotic pressure or unfavorable habitat conditions (Al-Yemeni \& Sher, 2010). This is 
in accordance with the findings of this study. Nazir \& Malik (2006) reported nanophanerophyte and therophytes as the biological spectra of Sarsawa hill Kotli. In addition, hot, dry and waterlogged conditions combined with overgrazing resulted in harsh conditions within the grove. This also agrees with the reports by Sher \& Khan (2007), as well as Amjad et al. (2017), and Abbas et al. (2020) stating therophytes and nanophanerophytes are characteristic of subtropical ecosystems. Structurally and floristically, sub-tropical dry forests are less complex than wet forests, comprising about half or less wet forest tree species (Hasnat \& Hossain, 2020). The forests serve as a refuge for valuable and endangered plants and animals. Hence, further study is required to quantify the data and suggest plans to conserve the sacred groves.

Table 4. Life-form analysis with different leaf size.

\begin{tabular}{|c|c|c|c|c|c|c|c|c|c|}
\hline \multirow{2}{*}{ Raunkiaer's life form } & \multicolumn{8}{|c|}{ Leaf spectra } & \multirow{2}{*}{ Total } \\
\hline & $\mathrm{Ap}$ & Le & $\mathrm{Na}$ & $\mathrm{Mi}$ & No & $\mathrm{Me}$ & $\mathrm{Ma}$ & $\mathrm{Mg}$ & \\
\hline Phanerophytes & 1 & 2 & 3 & 9 & 18 & 32 & 9 & 2 & 76 \\
\hline Megaphanerophytes & & & & 1 & & 1 & 3 & & 5 \\
\hline Mesophanerophytes & & & 1 & & 2 & 2 & 2 & & 7 \\
\hline Nanophanerophytes & 1 & 2 & 2 & 8 & 16 & 29 & 4 & 2 & 64 \\
\hline Chamaephytes & & 1 & 2 & 1 & 10 & 7 & 10 & 1 & 32 \\
\hline Hemicryptophytes & & 17 & 10 & 3 & & & & & 30 \\
\hline Cryptophytes & & 1 & 1 & & & 2 & 1 & & 5 \\
\hline Therophytes & & 6 & 17 & 22 & 18 & 8 & 3 & & 74 \\
\hline Total & 1 & 27 & 33 & 35 & 46 & 49 & 23 & 3 & 217 \\
\hline
\end{tabular}

Notes: Ap= Aphyllous; Le= Leptophyll; Ma= Macrophyll; Me= Mesophyll; Mg= Megaphyll; Mi= Microphyll; Na= Nanophyll; No= Notophyll

Leaf spectra. According to the overall leaf size spectrum (Table 1, Table 4, Fig. 4), there were 27 leptophyllous (12.44\%), 33 nanophyllous (15.21\%), 35 microphyllous (16.13\%), 46 notophyllous (21.20\%), 49 mesophyllous (22.58\%), 23 macrophyllous $(10.60 \%)$ and 3 megaphyllous (1.38\%), in the sacred grove. However, Cuscuta reflexa was an aphyllous $(0.46 \%)$ species. Meanwhile, 11 Poaceae $(2.67 \%)$, nine Poaceae $(2.67 \%)$, eight
Fabaceae $(2.86 \%)$, six Malvaceae (1.71\%), six Cucurbitaceae $(1.52 \%)$ and five Malvaceae $(1.33 \%)$ were discovered to be dominant families of the phylums leptophyll, nanophyll, microphyll, notophyll, mesophyll and macrophyll respectively. Table 1 and Table 4, as well as Fig. 4, also show the phylum megaphyll is equally distributed into Araceae, Cannabaceae and Fabaceae families.

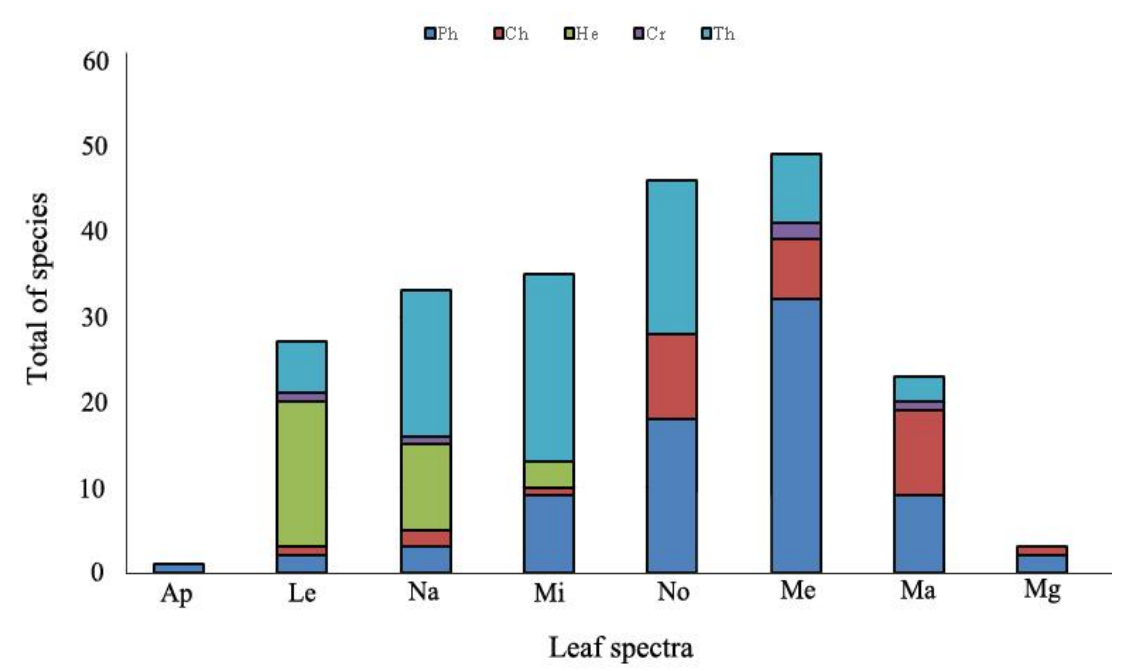

Fig. 4. Leaf spectral variation $(\mathrm{Ap}=$ Aphyllous; $\mathrm{Le}=$ Leptophyll; $\mathrm{Ma}=\mathrm{Macrophyll} ; \mathrm{Me}=\mathrm{Mesophyll} ; \mathrm{Mg}=\mathrm{Megaphyll}$; $\mathrm{Mi}=$ Microphyll; $\mathrm{Na}=$ Nanophyll; $\mathrm{No}=$ Notophyll; $\mathrm{Ph}=$ Phanerophytes; $\mathrm{Ch}=$ Chamaephytes; $\mathrm{He}=\mathrm{Hemicryptophytes} ; \mathrm{Cr}=$ Cryptophytes; $\mathrm{Th}=$ Therophytes). 
A leaf spectrum describes the plant adaptation and association in a community, with small-sized leaves present at the base and large leaves at the top, and is also correlated with climatic warming and water availability in the soil (Weber et al., 2012; Sakschewski et al., 2015; Badshah et al., 2016). The presence of middle-sized leaves indicates a sub-tropical climate. Furthermore, the meso and notophyllous elements dominant in the sacred grove, show moisture availability or wet condition. As a result of the therophytes and chamaephytes present, the proportion of leaf size groups was observed to change seasonally. However, in all seasons, the phanerophytes and some chamaephytes retained almost the same status.

IUCN categorization. About 161 of these 217 plants have currently not been evaluated. Furthermore, there are 55 Least Concerned (LC) and 1 Vulnerable (VU) species. Table 1 shows the IUCN categorization. Based on this grouping, climber Cayratia pedata is the vulnerable species (Saha et al., 2015). The results of threatened species assessment show most of the flora are perennial trees, while the phytosociological study with ecological data on IUCN red listed plants, indicates the plants are currently present and regenerate in the sacred groves, but disappear in nearby forests. This study therefore highlights the status and distribution of these species within the study area, the ecological characteristics required for their survival, and the threats to some of the species, identified by the IUCN criteria. Numerous factors led to the rise in numbers of vulnerable species. Overgrazing has been a significant cause of seedling destruction. However, anthropogenic activities, including the development of the plant and land-use amendment, are the foremost vital cause of this deterioration.

\section{CONCLUSION}

This study shows the possibility of using the Raunkiaer strategy to evaluate the major differences between the populations of angiosperm plants, associations in the forested landscape or biome, the percentage of flora species resulting from the established ecological parameters and environmental gradients. In addition, life-form analysis clearly shows the sacred grove's biological spectrum is "thero-chamaephytic" phytoclimate. Meanwhile, there is a need to further juxtapose the adjacent natural strands pattern along the environmental gradients, to reveal more than the mere forest covers in the ecosystem data. This indicates biotic variables are significant in shaping landscape vegetation, by guiding succession, and demonstrates the effect of anthropogenic diseases favoring therophyte growth, within the sacred grove. Therefore, any more damage to the sacred grove is able to facilitate potential changes within its present phytoclimate.

\section{ACKNOWLEDGEMENTS}

Special thanks to all informants who generously shared their knowledge of local plants and traditional medicinal uses. We are grateful for the proper field identification of plant species by Dr. G. G. Maity, ex-professor at Kalyani University.

\section{REFERENCES}

Abbas AM, Al-Kahtani MA, Alfaifi MY, Elbehairi SEI, Badry MO. 2020. Floristic diversity and phytogeography of Jabal Fayfa: A subtropical dry zone, South-West Saudi Arabia. Diversity. vol 12(9): 1-20. doi: https://doi.org/10.3390/d12090345.

Al-Yemeni M, Sher H. 2010. Biological spectrum with some other ecological attributes of the flora and vegetation of the Asir Mountain of South West, Saudi Arabia. African Journal of Biotechnology. vol 9(34): 5550-5559.

Amjad MS, Arshad M, Page S, Qureshi R, Mirza SN. 2017. Floristic composition, biological spectrum and phenological pattern of vegetation in the subtropical forest of Kotli District, AJK, Pakistan. Pure and Applied Biology (PAB). vol 6(2): 426447.

doi: http://dx.doi.org/10.19045/bspab.2017.60043.

Anderson T. 1862. Letter from Thomas Anderson to Sir Joseph Dalton Hooker. Richmond: Royal Botanic Gardens, Kew. $\quad$ p 6. https://plants.jstor.org/stable/10.5555/al.ap.visual.k dcas6139.

Asif M, Iqbal Z, Alam J, Majid A, Ijaz F, Ali N, Rahman IU, Hussain S, Khan A, Qadir G. 2020. Floristic inventory and biological spectra of Balakot, District Mansehra, Pakistan. Acta Ecologica Sinica. vol 40(3): 197-203. doi: https://doi.org/10.1016/j.chnaes.2019.05.009. 
Badshah L, Hussain F, Sher Z. 2016. Floristic inventory, ecological characteristics and biological spectrum of plants of Parachinar, Kurram agency, Pakistan. Pakistan Journal of Botany. vol 48(4): 1547-1558.

Berkes F. 2017. Sacred ecology. $4^{\text {th }}$ ed. New York: Routledge. $\quad \mathrm{p} \quad 394 . \quad$ doi: https://doi.org/10.4324/9781315114644.

Bhagwat SA, Rutte C. 2006. Sacred groves: Potential for biodiversity management. Frontiers in Ecology and the Environment. vol 4(10): 519-524.

Chase MW, Christenhusz MJM, Fay MF, Byng JW, Judd WS, Soltis DE, Mabberley DJ, Sennikov AN, Soltis PS, Stevens PF. 2016. An update of the Angiosperm Phylogeny Group classification for the orders and families of flowering plants: APG IV. Botanical Journal of Lineanean Society. vol 181: 1-20. doi: https://doi.org/10.1111/boj.12385.

Datta SC, Majumdar NC. 1966. Flora of Calcutta and vicinity. Bulletin of Botanical Society of Bengal. vol 20: 6-120.

de Paula LFA, Mota NFO, Viana PL, Stehmann JR. 2017. Floristic and ecological characterization of habitat types on an inselberg in Minas Gerais, southeastern Brazil. Acta Botanica Brasilica. vol 31(2): 199-211. doi: $\quad$ https://doi.org/10.1590/0102$33062016 a b b 0409$.

Farooq M, Anjum W, Hussain M, Saqib Z, Khan KR, Shah AH, Gul S, Jabeen S. 2019. Forest situation analysis and future forecasting of famous Upper Tanawal forests ecosystems on western banks of lesser Himalaya. Acta Ecologica Sinica. vol 39(1): 9-13. https://doi.org/10.1016/j.chnaes.2018.07.003.

Gastauer M, Neto M, Alves JA. 2017. Updated angiosperm family tree for analyzing phylogenetic diversity and community structure. Acta Botanica Brasilica. vol 31(2): 191-198. doi: https://doi.org/10.1590/0102-33062016abb0306.

Ghildiyal JC, Barthwal N, Khantwal A. 2016. Life-forms and biological spectrum of Deeva Ka Daanda a sacred grove in Garhwal Himalaya. Indian Forester. vol 142(2): 167-178.

Gnanasekaran G, Nehru P, Narasimhan D. 2012. Angiosperms of Sendirakillai sacred grove (SSG), Cuddalore district, Tamil Nadu, India. Check List. vol 8(1): 113-129. doi: https://doi.org/10.15560/8.1.113.

Haines HH. 1926. The Botany of Bihar and Orissa: an account of all the known indigenous plants of the province and of the most important or most commonly cultivated exotic ones. Nature. vol 117: 225-226. doi: https://doi.org/10.1038/117225b0.

Haq SM, Malik AH, Khuroo AA, Rashid I. 2018. Floristic composition and biological spectrum of Keran-a remote valley of northwestern Himalaya. Acta Ecologica Sinica. vol 39(5): 372-379. doi: https://doi.org/10.1016/j.chnaes.2018.12.001.

Hasnat GNT, Hossain MK. 2020. Global overview of tropical dry forests. In: Bhadouria R, Tripathi S, Srivastava P, Singh P (eds). Handbook of research on the conservation and restoration of tropical dry forests. Pennsylvania: IGI Global. pp 1-23.

Hooker JD. 1875. The flora of British India. Nature. vol 12: 3-5. doi: https://doi.org/10.1038/012003a0.

Hughes JD, Chandran MDS. 1998. Sacred groves around the earth: An overview. In: Ramakrishnan PS, Saxena KG, Chandrashekara UM (eds). Conserving the sacred for biodiversity management. New Delhi: Oxford and IBH Publishing Co. pp 69-86.

Jain SK, Rao RR. 2016. A Handbook of field and herbarium methods. New Delhi: Today and Tomorrow's Printers and Publishers. pp 1-157.

Kent EF. 2013. Sacred groves and local gods: religion and environmentalism in South India. New Delhi: Oxford University Press.

Khan ML, Khumbongmayum AD, Tripathi RS. 2008. The sacred groves and their significance in conserving biodiversity: An overview. International Journal of Ecology and Environmental Sciences. vol 34(3): 277-291.

Khan W, Khan SM, Ahmad H, Alqarawi AA, Shah GM, Hussain M, Abd-Allah EF. 2018. Life forms, leaf size spectrum, regeneration capacity and diversity of plant species grown in the Thandiani forests, district Abbottabad, Khyber Pakhtunkhwa, Pakistan. Saudi Journal of Biological Science. vol 25(1): 94-100. doi: https://doi.org/10.1016/j.sjbs.2016.11.009.

Mitra JN. 1958 Flowering Plants of Eastern India: Monocotyledons, Volume 1. Kolkata: The World Press Private Ltd. p 388.

Muller-Dombois D, Ellenberg H. 1974. Aims and methods of vegetation ecology. New Jersey: John Wiley \& Sons. pp 10-22.

Nazir A, Malik ZH. 2006. Life form and index of similarity of plant communities recorded at Sarsawa Hill District Kotri. Journal of Research in Science Teaching. vol 17(1): 27-33.

Paria N. 2005. Medicinal plant resources of south West Bengal (Vol. 1). Kolkata: Research Wing, Directorate of Forests in collaboration with Department of Environment, Government of West Bengal. pp 1-198.

Paria ND. 2010. Medicinal plant resources of south West Bengal Vol II. Kolkata: Research Wing, Directorate of Forests, Government of West Bengal, in collaboration with Department of Environment, Government of West Bengal. pp 1-124.

Pérez-Luque AJ, Bonet FJ, Pérez-Pérez R, Aspizua R, Lorite J, Zamora R. 2014. Sinfonevada: dataset of floristic diversity in Sierra Nevada forests (SE Spain). PhytoKeys. vol 35: 1-15. doi: https://doi.org/10.3897/phytokeys.35.6363.

Prain D. 1903. Bengal plants vol. 2. Kolkata: Botanical Survey of India. doi: https://doi.org/10.5962/bhl.title.50150.

Rath S, Ormsby AA. 2020. Conservation through Traditional Knowledge: A Review of Research on the Sacred Groves of Odisha, India. Human 
Ecology. vol 48(4): 455-463. doi: https://doi.org/10.1007/s10745-020-00173-1.

Raunkiaer C. 1934. The life forms of plants and statistical plant geography. Oxford: The Clarendon Press. pp $1-632$.

Reddy CS. 2008. Catalogue of invasive alien flora of India. Life Science Journal. vol 5(2): 85-87.

Saha D, Ved D, Ravikumar K, Haridasan K. 2015. Cayratia pedata. The IUCN Red List of Threatened Species 2015: e.T50126571A50131290. https://www.iucnredlist.org/. doi: https://dx.doi.org/10.2305/IUCN.UK.20152.RLTS.T50126571A50131290.en.

Sakschewski B, von Bloh W, Boit A, Rammig A, Kattge J, Poorter L, Peñuelas J, Thonicke K. 2015. Leaf and stem economics spectra drive diversity of functional plant traits in a dynamic global vegetation model. Global Change Biology. vol 21(7): 2711-2725. doi: https://doi.org/10.1111/gcb.12870.

Sen UK. 2018. Assessing the social, ecological and economic impact on conservation activities within human-modified landscapes: A case study in Jhargram District of West Bengal, India. International Journal of Conservation Science. vol 9(2): 319-336.

Sen UK, Bhakat RK. 2019. Floristic composition, biological spectrum and conservation status of a sacred grove from Jhargram district, West Bengal, India. Indian Forester. vol 142(2): 161-172.
Sharma J, Raina AK. 2017. Lifeform classification and biological spectrum of Nandini wildlife sanctuary, Jammu, J \& K, India. Environmental Conservation. vol 18(1\&2): 231-237. doi: https://doi.org/10.36953/ECJ.2017.181231.

Sher Z, Khan ZU. 2007. Floristic composition, life form and leaf spectrum of the vegetation of Chagharzai valley, district Buner. Pakistan Journal of Plant Science. vol 13(1): 57-66.

Verschuuren B, Wild R, Oviedo G, Mcneely J (eds). 2012. Sacred natural sites: Conserving nature and culture. London: Earthscan. p 300.

WBPSPM. 2011. District human development report: Paschim Medinipur. Kolkata: Department of Planning \& Statistics, Government of West Bengal. pp 1-283. http://www.wbpspm.gov.in/.

WCVP. 2020. World checklist of vascular plants, version 2.0. Richmond: Royal Botanic Gardens, Kew. http://wcvp.science.kew.org/.

Weber VS, Araus JL, Cairns JE, Sanchez C, Melchinger AE, Orsini E. 2012. Prediction of grain yield using reflectance spectra of canopy and leaves in maize plants grown under different water regimes. Field Crops Research. vol 128: 82-90. doi: https://doi.org/10.1016/j.fcr.2011.12.016.

Xu X, Zhang H, Xie T, Yue J, Zhao L, Tian Y. 2018. Geographical patterns of Raunkiaerian life-form spectrum in China. Pakistan Journal of Botany. vol 50(4): 1509-1516. 